\title{
Spatial orientation of a limb using egocentric reference points
}

\author{
DOUGLAS D. LARISH \\ University of Iowa, Iowa City, Iowa \\ and \\ GEORGE E. STELMACH \\ University of Wisconsin, Madison, Wisconsin
}

\begin{abstract}
Testing the hypothesis that spatial localization can be based on an abstracted spatial location code, rather than on stored proprioceptive information, orientation of an unseen limb was contrasted under intra- and interlimb-movement conditions. In Experiment 1, movements were executed in the midline either vertically upward or horizontally forward in the sagittal plane. These results revealed that intralimb errors were smaller than interlimb errors only at the most distant criterion spatial targets, and it was hypothesized that positioning of a limb could be mediated by a spatial location code if spatial targets were coded in association with body reference points. Experiment 2 tested the egocentric referent hypothesis further by manipulating the availability of body-based spatial reference points under intra- and interlimb conditions. At spatial targets that could be coded in conjunction with body reference points, no difference was found between intra- and interlimb accuracy. In contrast, at spatial targets where body reference points were absent, or at least made difficult to rely on, accuracy was greater in the intralimb condition. It was concluded that spatial reference points, in this instance body-based, are necessary if the spatial positioning of a limb is to be based on the spatial location code. The data were also discussed within a more comprehensive framework of spatial frames of reference.
\end{abstract}

The complex faculty of spatial orientation in humans has interested psychologists for many years (Hollingworth, 1909; Holmes, 1919; Riddoch, 1917; Woodworth, 1899). This interest has been especially keen in the area of motor behavior, in which the relationship between orientation and action is an important one. An aspect of this relationship that has attracted considerable attention is the role of the socalled spatial reference system (Lashley, 1951) in the perception and localization of spatial targets (Allen, Siegel, \& Rosinski, 1978; Attneave \& Benson, 1968; Gross, Webb, \& Melzack, 1974; Howard \& Templeton, 1966; Luria, 1966; MacNeilage, 1970; Paillard \& Brouchon, 1968; Pick, 1970; Russell, 1976; Wallace, 1977). The spatial reference system is proposed to be

\footnotetext{
The present research was supported by grants from the Research committee of the Graduate School, University of WisconsinMadison, Project No. 190400, Biomedical Research Support Grants 144-6805 and 144-J432, and Air Force Grant AFOSR-78-3691 awarded to G. E. Stelmach. We would like to thank $M$. Teghtsoonian for her helpful comments in the revision of this paper. Requests for reprints should be sent to George E. Stelmach, Motor Behavior Laboratory, 2000 Observatory Drive, University of Wisconsin, Madison, Wisconsin 53706, or to Douglas D. Larish, Motor Behavior Laboratory, S 301 Fieldhouse, University of lowa, Iowa City, Iowa 52242.
}

a representation of physical space that is defined primarily by a set of interrelated reference points. Accordingly, a spatial target is perceived and coded in relation to known reference points, and, when reorientation to this target is required, the constructed relationship between the reference points and the target is thought to guide this action. Two generally recognized categories of spatial reference systems are the egocentric and the allocentric (Howard \& Templeton, 1966; Pick, 1970; Rieser \& Pick, 1976). In an egocentric system, orienting actions are based on body-related reference points, whereas, in an allocentric system, they are based on environment-related reference points.

MacNeilage (1970) was one of the first motor control theorists who stressed the importance and necessity of a three-dimensional spatial reference system for movement production. In contrast to the more traditional views of movement regulation, for example, Adams's (1971) closed-loop theory, MacNeilage's target hypothesis maintains that the achievement of a desired spatial position is not accomplished via the storage of sensory consequences from preliminary movements. Rather, the initial sensory information is transformed into a spatial location code, with the spatial reference system assisting 
in the transformation process. When relocating this target is desired, one simply determines the current spatial position, retrieves from memory the position's spatial "coordinates," and generates from these two pieces of information the motor commands required to execute the intended action.

Although MacNeilage's (1970) original ideas were developed as an explanation of speech production and speech control, they have since been extended to include movements of one's limbs (Morasso, 1981; Russell, 1976; Wallace, 1977). Irrespective of the musculature, however, if the spatial location code and the spatial reference system are to provide a general mechanism for movement control, it must be shown that (1) the spatial location code can be accessed in memory independently of movements responsible for its initial storage (Russell, 1976), and (2) the presence (or absence) of spatial reference points is a principle determinant of orienting performance.

Wallace (1977) reported evidence that accurate limb orientation can be accomplished independently of stored proprioceptive inputs. Subjects were required to position a limb to an experimenter-defined spatial target under two conditions. A criterion and subsequent reproduction movement were performed either with the same limb (e.g., right-right) or with opposite limbs (e.g., right-left). In the intralimb condition, stored proprioceptive inputs experienced during the criterion movement were available to guide the reproduction movement. In the interlimb condition, however, Wallace argued that it would be difficult to use directly proprioceptive inputs of the spatial location gained during the criterion movement for reproduction purposes. Therefore, if accurate reproduction were maintained, localization must have been based on some alternate form of information, namely, the spatial location code. In an initial experiment, in which a criterion spatial target was approached from opposite directions on interlimb trials, reproduction errors were significantly smaller in the intralimb condition. When, however, direction of approach was held constant, the superiority of the intralimb condition was nullified. Such a result supports the hypothesis that a spatial location can guide movement reproduction independently of stored proprioceptive information, although its generality may be questioned. The first experiment in the present study further evaluated the ability of the spatial location code to mediate accurate limb localization by contrasting intra- and interlimb movements that were executed vertically upward or horizontally forward in the sagittal plane. ${ }^{1}$

Another question of interest, and one that has yet to be addressed, is: How important are spatial reference points to the development of a precise and robust spatial location code? As stated earlier, the spatial location code has been hypothesized to be represented in memory as a point within a three-dimensional reference system (MacNeilage, 1970; Russell, 1976). And, since reference points are thought to be an integral component of spatial reference systems (Hart \& Moore, 1973; Pick, Yonas, \& Rieser, 1979; Sadalla, Burroughs, \& Staplin, 1980), it is reasonable to assume that their availability will directly influence the development of the spatial location code. More specifically, the knowledge derived from spatial reference points may be necessary to construct a spatial location code that can sustain accurate orienting performance, and when such knowledge is absent, a less precise spatial location code will develop, resulting in an associated decrement in orienting performance. Results from Experiment 1 suggested that this may indeed be the case, and a second experiment was designed to examine such a possibility.

\section{EXPERIMENT 1}

If specific proprioceptive information regarding the criterion spatial target is required for movement reproduction, then reproduction errors in the intralimb condition should be smaller than in the interlimb condition, independently of target location. Alternatively, if the spatial location code can mediate movement reproduction, then reproduction errors in the intra- and interlimb conditions should be equivalent at each target location.

\section{Method}

Subjects. Twelve ( 7 males, 5 females) right-handed subjects performed the vertically upward movements and 11 (3 males, 8 females) right-handed subjects performed the horizontally forward movements. The participants were recruited from the University of Wisconsin-Madison student population and were between 18 and 32 years of age.

Apparatus. In the vertical task, a 2 -cm-diam thimble was affixed to a metal rod $90 \mathrm{~cm}$ high and $1.3 \mathrm{~cm}$ in diameter. The thimble could be easily moved along the rod, and, when released, its final position was maintained by a counterbalance weight attached via a pulley system. In the horizontal task, the stylus arm of a Numonics Graphics Digitizer (Model 1224) was constrained to move in one dimension by a $70-\mathrm{cm}$-long and .5 - $\mathrm{cm}$-wide track. To preclude visual and auditory inputs, the subjects wore black opaque goggles and headphones.

Procedure. After entering the testing chamber, a subject was seated so that his or her midline was directly in front of the movement track and the movement range could be completed by both arms. A familiarization period followed, during which the reproduction conditions and verbal commands were explained. Each trial began with a verbal command that told the subject the limb to be used during the criterion movement. A second command cued the beginning of the criterion movement. After contacting a mechanical stop, the subject maintained the criterion spatial location for $2 \mathrm{sec}$ and then returned the arm to the start position area. During the 2-sec location duration, each subject was instructed to rehearse the target's spatial position; however, no specific instructions for rehearsal were given. The experimenter returned the thimble or stylus to the original start position, which remained constant throughout the experiment, and then issued the reproduction command for the same or opposite limb. In the vertical task, the start position was at the bottom of the movement range, and in the horizontal task, the start position was at the near-point of 
the movement range. The subjects were further instructed to make the reproduction movement smoothly and directly to the preestablished location, avoiding subsequent adjustments once the movement was terminated.

On the intralimb trials, the criterion and reproduction movements were executed with the right arm; on interlimb trials, the criterion movement was executed with the right arm, but the reproduction movement was executed with the left arm. ${ }^{2}$ For the vertical movements, targets were set at $10,25,35,50$, and $60 \mathrm{~cm}$ from the start position, and for the horizontal movements, targets were set at $10,20,30,40$, and $50 \mathrm{~cm}$ from the start position. Due to limitations in horizontal reach, it was not possible to maintain identical movement lengths in both instances. A testing session consisted of 30 intralimb and 30 interlimb trials, which were further partitioned into six replicates per criterion location. Trials were presented randomly, so that subjects did not know the limb of reproduction prior to the reproduction movement.

\section{Results and Discussion}

Localization errors at each target were recorded to the nearest millimeter, and absolute error (AE), constant error (CE), and variable error (VE) were calculated. $\mathrm{AE}$ is the absolute (unsigned) deviation of the reproduction spatial target from the criterion target and represents an estimate of general movement accuracy. CE is the signed deviation of the reproduction spatial target from the criterion target and is regarded as an index of movement bias: the degree of undershooting (negative score) and overshooting (positive score) around the criterion target. VE is the standard deviation of the signed scores around their $\mathrm{CE}$ mean and is an estimate of the variability or precision of movement. For a more detailed description of the derivation and interpretation of these error measures, see Safrit, Spray, and Diewert (1980). Within each movement plane, the data were analyzed via a repeated measures analysis of variance, subjects (12 or 11) $\times \operatorname{limb}(2) \times$ target (5).

Vertically upward movements. The AE analysis indicated that only the limb main effect $[F(1,11)=$ $12.22, \mathrm{p}<.05]$ and the limb $\times$ target interaction $[F(4,44)=15.92, p<.05]$ were significant. A post hoc analysis of the interaction was carried out (using
Tukey's HSD procedure) in which inter- and intralimb reproduction accuracy were compared at each of the five criterion targets. This analysis showed that, at the $50-$ and $60-\mathrm{cm}$ targets, intralimb reproduction was more accurate than interlimb reproduction (see Table 1). Similar comparisons at the remaining targets were nonsignificant. For $\mathrm{CE}$, both the limb and target main effects were significant $[F(1,11)=6.53$ and $F(4,44)=13.99$, respectively; ps $<.05$ in each case]. The interaction of limb and target was also significant $[F(4,44)=4.64, p<.05]$ : Subsequent analysis showed that, at the $25-\mathrm{cm}$ target, greater overshooting was observed in the intralimb condition than in the interlimb condition, and at the 50- and $60-\mathrm{cm}$ targets, overshooting was found in the intralimb condition but undershooting was found in the interlimb condition. In VE, the limb and the target main effects were again significant $[F(1,11)=5.68$ and $F(4,44)=5.65 ; \mathrm{ps}<.05]$, as was the limb $\times$ target interaction $[F(4,44)=17.28, p<.05]$. Post hoc analysis of this interaction showed that intralimb reproduction was less variable than interlimb reproduction at the $60-\mathrm{cm}$ target. The remaining pairwise comparisons were nonsignificant.

Horizontally forward movements. The AE analysis revealed that the limb main effect and the limbtarget interaction were significant $[F(1,10)=5.67$ and $\mathrm{F}(4,40)=7.96$, respectively; ps $<.05]$. Further analysis of the interaction showed that intralimb reproduction was more accurate than interlimb reproduction at the 40 - and $50-\mathrm{cm}$ targets, while no other comparisons were significant (see Table 2). Analysis of CE showed that both main effects were significant $[\operatorname{limb}, F(1,10)=14.90$, and target, $F(4,40)=47.31$; ps <.05]. However, the limb $\times$ target interaction was also significant $[F(4,40)=6.76, p<.05]$. The post hoc analysis yielded four significant comparisons: At the $20-\mathrm{cm}$ target, the intralimb condition had larger overshooting errors than the interlimb condition, and at the 40 - and 50 -cm targets, the inter-

Table 1

Reproduction Accuracy (in Centimeters) of the Intra- and Interlimb Conditions at Each Criterion Spatial Target for the Vertically Upward Movements

\begin{tabular}{|c|c|c|c|c|c|c|c|c|c|c|}
\hline & \multicolumn{10}{|c|}{ Location } \\
\hline & \multicolumn{2}{|c|}{10} & \multicolumn{2}{|c|}{25} & \multicolumn{2}{|c|}{35} & \multicolumn{2}{|c|}{50} & \multicolumn{2}{|c|}{60} \\
\hline & Mean & SD & Mean & SD & Mean & SD & Mean & SD & Mean & SD \\
\hline \multicolumn{11}{|c|}{ Intralimb } \\
\hline $\begin{array}{l}A E \\
C E \\
V E\end{array}$ & $\begin{array}{l}2.10 \\
1.93 \\
1.50\end{array}$ & $\begin{array}{r}1.71 \\
1.90 \\
.76\end{array}$ & $\begin{array}{l}2.62 \\
2.10 \\
2.62\end{array}$ & $\begin{array}{l}2.59 \\
3.03 \\
1.55\end{array}$ & $\begin{array}{l}3.38 \\
2.13 \\
2.95\end{array}$ & $\begin{array}{l}2.98 \\
3.97 \\
1.48\end{array}$ & $\begin{array}{r}2.53 \\
.65 \\
2.58\end{array}$ & $\begin{array}{r}2.00 \\
3.16 \\
.51\end{array}$ & $\begin{array}{r}1.85 \\
.20 \\
1.69\end{array}$ & $\begin{array}{l}1.50 \\
2.37 \\
1.54\end{array}$ \\
\hline \multicolumn{11}{|c|}{ Interlimb } \\
\hline $\begin{array}{l}A E \\
C E \\
V E\end{array}$ & $\begin{array}{l}2.54 \\
2.18 \\
1.60\end{array}$ & $\begin{array}{r}2.05 \\
2.43 \\
.62\end{array}$ & $\begin{array}{r}2.53 \\
.69 \\
2.24\end{array}$ & $\begin{array}{r}2.76 \\
3.69 \\
.50\end{array}$ & $\begin{array}{l}3.49 \\
1.82 \\
3.21\end{array}$ & $\begin{array}{l}3.25 \\
4.41 \\
1.34\end{array}$ & $\begin{array}{r}3.49 \\
-2.00 \\
2.60\end{array}$ & $\begin{array}{l}2.77 \\
3.99 \\
1.33\end{array}$ & $\begin{array}{r}3.79 \\
-1.35 \\
3.51\end{array}$ & $\begin{array}{l}2.76 \\
4.49 \\
1.75\end{array}$ \\
\hline
\end{tabular}


Table 2

Reproduction Accuracy (in Centimeters) of the Intra- and Interlimb Conditions at Each Criterion Spatial Target for the Horizontally Forward Movements

\begin{tabular}{|c|c|c|c|c|c|c|c|c|c|c|}
\hline & \multicolumn{10}{|c|}{ Location } \\
\hline & \multicolumn{2}{|c|}{10} & \multicolumn{2}{|c|}{20} & \multicolumn{2}{|c|}{30} & \multicolumn{2}{|c|}{40} & \multicolumn{2}{|c|}{50} \\
\hline & Mean & SD & Mean & SD & Mean & SD & Mean & SD & Mean & SD \\
\hline \multicolumn{11}{|c|}{ Intralimb } \\
\hline $\mathrm{AE}$ & 2.81 & 1.95 & 3.98 & 2.48 & 2.73 & 2.11 & 1.89 & 1.47 & 1.65 & 1.57 \\
\hline $\mathrm{CE}$ & 2.25 & 2.28 & 3.71 & 2.87 & 1.15 & 3.25 & -.65 & 2.30 & -.44 & 2.24 \\
\hline $\mathrm{VE}$ & 1.28 & .66 & 1.83 & .43 & 2.21 & 1.01 & 1.74 & .85 & 1.51 & .86 \\
\hline \multicolumn{11}{|c|}{ Interlimb } \\
\hline $\mathrm{AE}$ & 3.52 & 2.14 & 2.98 & 1.93 & 2.43 & 1.98 & 4.01 & 3.18 & 4.02 & 2.61 \\
\hline $\mathrm{CE}$ & 3.29 & 2.48 & 2.15 & 2.73 & -.70 & 3.06 & -3.08 & 4.09 & -3.31 & 3.46 \\
\hline VE & 1.76 & .79 & 2.04 & .70 & 2.05 & .86 & 2.54 & 1.39 & 2.36 & 1.02 \\
\hline
\end{tabular}

limb condition had larger undershooting errors than the intralimb condition. Although the comparison at the $30-\mathrm{cm}$ target was also significant, it is difficult to assess its meaningfulness, because the positive magnitude of intralimb errors is nearly equivalent to the negative magnitude of interlimb errors. In fact, if the analysis were to be done on the unsigned means, the difference would be nonsignificant. For VE, the limb and target main effects were significant $[F(1,10)$ $=5.13$ and $F(4,40)=2.87$, respectively; ps $<.05]$. The limb by target interaction was also significant $[F(4,40)=2.63, p<.05]$, and post hoc analysis showed that intralimb reproduction was less variable than interlimb reproduction at the $40-$ and $50-\mathrm{cm}$ targets. Reproduction errors at the remaining targets were equivalent.

From the outset, it was thought that localization errors in the interlimb condition would be either equivalent to or greater than localization errors in the intralimb condition at all spatial targets. The data fail to substantiate this expectation. Instead, they indicate that the intralimb condition is consistently more accurate and precise only at the two farthest targets, the two conditions being generally equivalent at the remaining targets. Furthermore, this pattern is seen for movements in the sagittal plane both vertically upward and horizontally forward. Consequently, these data, like Wallace's (1977), place some restrictions on the generality of control by the spatial location code.

How can such findings be explained within the context of the target hypothesis? The restrictions observed in the present experiment may be related to the availability and use of body-based spatial reference points. It might be that, when intra- and interlimb reproduction was equivalent, body reference points enhanced the perception and coding of these spatial targets; that is, body reference points provided supplementary spatial information that facilitated the transformation of the proprioceptive cues into a precise and well-defined spatial location code, which was, in turn, used effectively by the contralateral arm. In contrast, at the spatial targets where interlimb errors were larger than intralimb errors, the additional and perhaps necessary spatial information afforded by body reference points may have been lacking. Therefore, since it would have been based on a less precise spatial location code, reproduction by the contralateral arm was at a disadvantage. Such an interpretation is strengthened by the fact that 50 - and $60-\mathrm{cm}$ targets in the vertically upward direction were above the head, a position where body reference points are unavailable. This conclusion implies that, by itself, the transformation process is somewhat inefficient, so that during the transformation from the proprioceptive cues to the spatial location code some spatial information is lost. At the same time, such a loss seems to be averted or at least compensated for when spatial targets can be coded in conjunction with body reference points.

Assuming the midline is used as a body reference point in the horizontally forward movements, how is it possible that intra- and interlimb performance is equivalent at some targets in the midline, yet at other targets also in the midline, intralimb performance is more accurate than interlimb performance? Nelson and Chaiklin (1980) have recently reported particularly relevant data. These authors found that when the physical distance between to-be-remembered spatial targets and spatial reference points increases, spatial memory decreases. A similar idea has also been expressed by Pick, Yonas, and Rieser (1979). Although Nelson and Chaiklin's conclusions were based on a visual spatial-location task, this same phenomenon may be operating in the above instances. More specifically, for the farthest targets (where intralimb reproduction was more accurate and precise than interlimb reproduction), the midline may no longer be a salient spatial reference point. Hence, a less accurate spatial location code is developed, one which is unable to sustain spatial localization of the contralateral arm. 
There are also a number of other interesting findings in the present data that are for the most part consistent across the vertical and horizontal movements. For both $\mathrm{AE}$ and $\mathrm{VE}$, intralimb reproduction movements can be described as an inverted-U function of criterion target distance: Spatial errors increase at targets up to approximately $35 \mathrm{~cm}$ and then begin to decrease at targets beyond this point. A reduction in spatial errors at the more distant spatial targets might be viewed as an unusual finding, but this pattern has been repeatedly found in studies requiring movements in the same plane of the body as those reported here (Diewert, 1976; Larish, Stelmach, \& McCracken, 1979). Moreover, this result is consistent with neurophysiological evidence demonstrating that, as the limb approaches the extreme ranges of flexion and extension, joint receptor (Burgess \& Clark, 1969) and muscle spindle (Matthews, 1972) activity increases, thereby providing the basis for a more accurate assessment of movement reproduction than at intermediate ranges of movement.

A similar pattern, however, was not found in $\mathrm{AE}$ and VE for interlimb movements. Here the errors can be described as a roughly linear increasing function of criterion target distance. Such a finding is, of course, consistent with the hypothesized relationship between body reference points and the development of the spatial location code. Also, the fact that this pattern is quite different from that found in the intralimb condition further supports the contention that interlimb reproduction is based on movement information different in form from stored proprioceptive inputs.

The $C E$ data also yielded interesting findings. For interlimb movements, targets closest to the start position were overshot and those farthest were undershot. Such a pattern of errors has come to be known as the range effect and is a frequently observed finding in limb positioning studies. Note, however, that this range effect is not evident in the intralimb condition. Although closer targets are overshot, there is a lack of substantial undershooting at the farthest two targets. In fact, there is virtually no error at these targets. In addition, undershooting of the criterion target appears to occur at a much earlier point in the movement range for interlimb movements than it does for intralimb movements. The reason for these latter two findings is not altogether clear, but they do point out once again that two different types of movement information are regulating intra- and interlimb movements.

\section{EXPERIMENT 2}

The foregoing interpretations certainly raise questions about the relationship between spatial reference points and the spatial location code during orientation of an unseen limb. The purpose of this second experiment was to determine whether body-based reference points have a direct mediating effect on reproduction errors when localization must be based on the spatial location code. The intra/interlimb procedure was again used, but this time spatial targets in two-dimensional space were selected on the basis of their correspondence with body-based reference points. For the purposes of this experiment, a spatial target that fell within an area defined by the width of the shoulders and at a distance of no more than $30 \mathrm{~cm}$ was considered to be easily associated with at least one body referent. (The distance of $30 \mathrm{~cm}$ is based on the results from the horizontal movements in Experiment 1 and is only meant to be an estimate.) For instance, it was assumed that a target placed in front of the shoulder could be coded into memory, with the shoulder serving as a reference point. It was further assumed that it would be difficult to associate a target outside this range with a stationary body reference point. If these reference points are necessary for accurate limb positioning when reproduction must be based on the spatial location code, the expected pattern of results is rather straightforward: At locations where body reference points can be used to code the spatial target, intra- and interlimb errors should be equivalent, but at locations where body reference points are more difficult to use directly in coding the spatial target, errors in the interlimb condition should be greater than in the intralimb condition.

\section{Method}

Subjects. Twelve ( 9 males, 3 females) right-handed participants ranging in age from 18 to 30 years were recruited from the University of Wisconsin-Madison.

Apparatus. To allow movements in two dimensions, a Numonics Graphics Digitizer (Model 1224) was used. The digitizer was mounted on a Formica plate $(44 \times 82 \times 1.3 \mathrm{~cm})$, which in turn rested on a table $67 \mathrm{~cm}$ in height, so that movements were executed horizontally forward in the sagittal plane. The recording head was placed $60 \mathrm{~cm}$ from the base of the movement range so that a stylus arm could be easily moved to any position on the Formica plate. The nature of the movement task required that criterion movements be restricted to one dimension; this was accomplished by constraining the criterion movement within a .5 $\mathrm{cm}$-wide and $55-\mathrm{cm}$-long track, which was subsequently removed for the reproduction movement. The digitizer was interfaced with a Digital Corporation PDP-8e computer, which recorded the $x-y$ coordinates of the terminal position on a reproduction movement. To preclude visual input, the subjects wore black opaque goggles.

Procedure. The general procedures and instructions were in most respects like those of Experiment 1. Three criterion targets were placed so that they could be easily associated with a body referent point (see Figure 1): Target 2 corresponded to a point $20 \mathrm{~cm}$ in front of the midline and was chosen because it represented a location where intra- and interlimb accuracy were equivalent in the previous experiment. Targets 1 and 3 corresponded to locations $15 \mathrm{~cm}$ to the left and right of the midline and $20 \mathrm{~cm}$ from the base of the movement range at a straight-line distance of $26 \mathrm{~cm}$ from the start position. They were approximately in line with the shoulders and could be coded in conjunction with this body reference point. In addition, three targets were designated as outside the direct influence of body reference points. Target 5 was placed $40 \mathrm{~cm}$ in front of the midline, and it was the point at which intra- and interlimb performance first became dif- 


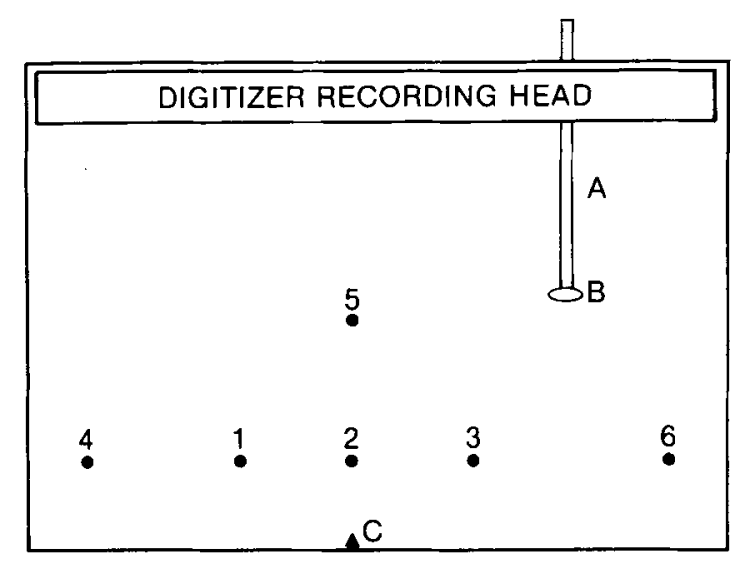

Figure 1. Diagram of the apparatus and work surface. Subjects were seated with the midline at $C$, which also served as a central starting position for all trials. Subjects grasped a small handle, B, at the distal end of the stylus arm, $C$. Since the stylus arm could move freely in two dimensions, the handle could be positioned to any of the six spatial targets. Targets 2 and 5 were positioned 20 and $40 \mathrm{~cm}$ from $C$. Targets 1 and 3 were positioned $15 \mathrm{~cm}$ to the left and right of the midline at a stmight line distance of $26 \mathrm{~cm}$ from $C$. Targets 4 and 6 were positioned $35 \mathrm{~cm}$ to the left and right of the midline at a straight line distance of $41 \mathrm{~cm}$ from $\mathrm{C}$.

ferentiated in the initial experiment. Targets 4 and 6 were placed $35 \mathrm{~cm}$ to the left and right of the midline, at a straight-line distance of $41 \mathrm{~cm}$ from the start position. With the arm at the side of the body, these targets were nearly $20 \mathrm{~cm}$ beyond each shoulder, and it was thought that it would be difficult to code these targets in direct relation to a stationary body referent point. Since data on movement accuracy in two dimensions for all movement combinations were unavailable, another change in procedure was the addition of the left-left and left-right limb combinations. Subjects performed a total of 72 trials that were partitioned into three replicates per limb combination at each of the six criterion targets. Trials were once again randomly presented.

\section{Results and Discussion}

The experimental design corresponded to a completely within-subjects $12 \times 4 \times 6$ (subjects $\times$ limb $x$ target) factorial, and the data analysis consisted of a repeated measures analysis of variance. The primary dependent measure was radial error, which was defined as the straight-line distance between the criterion spatial target and the terminal reproduction target. For movements in two dimensions, radial er- ror is a measure of the overall accuracy of movement reproduction.

The analysis of variance indicated that only the limb $\times$ target interaction was significant $[F(15,165)$ $=1.90, \mathrm{p}<.05$ ]. Post hoc comparisons were then made using Tukey's HSD procedure. To address the predictions stated earlier, reproduction errors between the right-right and left-right combinations and between the left-left and right-left combinations were compared as a function of spatial target. For the three criterion targets that could be coded in conjunction with body reference points $(1,2,3)$, only one of the six pairwise comparisons was significant: At target 1, right-right accuracy was better than leftright accuracy (see Table 3). The pattern of results was markedly different at the locations that were not directly associated with body reference points. At criterion targets 4,5 , and 6 , right-right errors were smaller than right-left errors. Similarly, left-left errors were smaller than right-left errors.

Of primary interest in Experiment 2 was the difference in effect of body reference points on interlimb and intralimb reproduction accuracy. Their accuracy was equivalent only at the three spatial targets directly codable with body reference points; at other targets intralimb accuracy was greater. Thus the data do indeed support the hypothesis that the availability of egocentric reference points facilitates localization based on a spatial reference code. Moreover, the results at targets 2 and 5 provide a partial replication of Experiment 1.

A series of post hoc comparisons were also conducted to determine if the two intralimb combinations (right-right and left-left) differed in accuracy at each criterion target. This analysis revealed that all comparisons were nonsignificant $(p>.05)$, indicating that reproduction accuracy between these two combinations was equivalent. An identical analysis was made on the interlimb combinations (left-right and right-left), and just one pairwise comparison was significant $(p<.05)$ : At location 4, right-left errors were smaller than left-right errors. Thus, reproduction accuracy in the two interlimb combinations was equivalent. A third post hoc analysis compared intraand interlimb errors at each target, but this time the

Table 3

Mean Radial Error (in Centimeters) of the Four Limb Combinations at Each Spatial Target

\begin{tabular}{|c|c|c|c|c|c|c|c|c|}
\hline \multirow[b]{3}{*}{ Location } & \multicolumn{8}{|c|}{ Limb } \\
\hline & \multicolumn{2}{|c|}{ RR } & \multicolumn{2}{|c|}{$\mathbf{L L}$} & \multicolumn{2}{|c|}{ RL } & \multicolumn{2}{|c|}{ LR } \\
\hline & Mean & SD & Mean & SD & Mean & SD & Mean & SD \\
\hline 1 & 4.10 & 1.53 & 5.18 & 1.54 & 5.70 & 1.68 & 5.63 & 1.89 \\
\hline 2 & 4.22 & 1.88 & 4.64 & 1.78 & 5.24 & 2.13 & 4.60 & 1.50 \\
\hline 3 & 4.77 & 1.88 & 5.37 & 1.84 & 5.31 & 2.56 & 5.02 & 1.84 \\
\hline 4 & 4.70 & 1.56 & 4.77 & 2.26 & 6.24 & 2.65 & 7.76 & 3.02 \\
\hline 5 & 3.25 & 1.44 & 3.57 & 1.94 & 6.12 & 2.93 & 5.61 & 2.68 \\
\hline 6 & 4.62 & 1.78 & 3.76 & 1.24 & 6.14 & 2.14 & 6.36 & 2.96 \\
\hline
\end{tabular}


limb of the criterion movement was kept constant (right-right vs. right-left and left-left vs. left-right). This analysis showed the same pattern of results as when the limb of the reproduction movement was kept constant. Given the results of these latter two analyses, limb dominance does not appear to influence the obtained results differentially. Such findings are consistent with those reported by Wallace (1977) and further argue against the view that in the previous experiment the intra- and interlimb manipulations were confounded with limb dominance.

\section{GENERAL DISCUSSION}

The present experiments were designed to investigate the conditions under which an abstract spatial location code (MacNeilage, 1970) can direct the accurate positioning of an unseen limb. A switched-limb technique (Wallace, 1977) was used to contrast conditions in which stored proprioceptive input was or was not available to regulate movement reproduction. Accurate limb positioning was achieved on the basis of a spatial location code, in general agreement with the previous findings of Buck (1979), Russell (1976), and Wallace (1977). Also, there was some indication that the nature of the information stored in the spatial location code is fundamentally different from stored proprioceptive information. In the intralimb condition, accuracy and precision errors are characterized by a curvilinear function; in the interlimb condition, they are characterized by a linear function.

An important restriction on the generality of control by the spatial location code also surfaced: Not all spatial positions are reproducible with equal accuracy and precision. We hypothesize that these differences are dependent upon spatial knowledge gained from body reference points. Only if body reference points provide salient spatial information by acting as organizing loci for other targets (Allen, Siegel, \& Rosinski, 1978; Sadalla, Burroughs, \& Staplin, 1980) is localization accomplished as proficiently with the abstracted spatial location code as with learned proprioceptive cues.

Similar results have also been found during visual search (Bremmer \& Bryant, 1977) and whole-body orientation in large-scale space (Acredolo, 1977, 1978). When reference points were absent, infants and children under 5 years of age used previously experienced sensory consequences (i.e., stored proprioceptive information) to localize a target. When, however, environmental reference points were introduced, a target was localized on the basis of its physical position (i.e., a spatial location code). Despite many differences between these studies and ours, a recurring finding is that salient spatial reference points allow one to regulate orienting actions via a spatial location code.
As operationally defined here, the designation of points between the shoulders and within $30 \mathrm{~cm}$ of the body as having readily available body reference points was somewhat arbitrary. A more precise specification of body reference points and the limits of their influence would benefit future experiments, but depends on expanding our current knowledge of the perception of egocentric space and its relation to physical space. It is likely, for example, that appropriate reference points vary as a function of the plane of the body and the type of movement. Also, we must determine the degree of correspondence between a perceived reference point and its actual physical position, as well as how this subjective-objective relationship affects orienting performance. For example, Wallace (1976) showed that some subjects are unable to position the limb accurately in line with a body referent, the midline. Although there appeared to be little within-subject variation, a considerable amount of between-subject variation was found. And it may be that those persons who perform poorly on such spatial tasks also have a poor perception of egocentric space and body reference points. If this is the case, it would be important to ascertain if these inaccuracies can be reduced or eliminated with training on the perception of body reference points.

Another potentially worthwhile research effort is to pursue the effects of allocentric reference points on limb orientation. They can certainly be identified more objectively and manipulated more readily than egocentric reference points. In fact, benefits in limb orientation have been reported in instances in which allocentric reference points were used, even when stored proprioceptive information was available. Both Shea (1977) and Winther and Thomas (1981) found that an objective, external frame of referencethe hour positions on the face of a clock-improved reproduction performance of an unseen limb. Shea also showed that, in the frame-of-reference group, spatial location information was retained in memory and used to guide movement reproduction accurately even after a 60 -sec retention interval, a remarkable finding considering that in similar experiments location information is retained for only approximately $20 \mathrm{sec}$.

Finally, it should be noted that our results may depend, not on the differential availability of body reference points, but rather on distortions in the alignment of body position. When the limb is reaching to the farther target positions, it nears full extension and causes some rotation of the upper body. In the intralimb condition, the rotation during the criterion and reproduction movements is always in the same direction, whereas, in the interlimb condition, it is in opposite directions. The closer targets do not induce this rotation. Thus, it may be that as long as the torso remains stationary there will be no decrement in the 
interlimb transfer of spatial location information, but that rotation in opposite directions effects such a decrement. Even if this were so, an explanation in terms of a spatial frame of reference is available. Rotation during the reproduction movement may alter the relationship between the spatial target and any body reference point that was being relied on, reducing the reliability of information about the target's position. The present study does not indicate which of the two interpretations is correct, but both raise interesting questions that, when answered, will add to our current understanding of spatial orientation within egocentric space.

\section{REFERENCES}

Acrebolo, L. P. Developmental changes in the ability to coordinate perspectives of a large-scale space. Developmental Psychology, 1977, 13, 1-8.

Acredolo, L. P. Development of spatial orientation in infancy. Developmental Psychology, 1978, 14, 224-234.

ADAms, J. A. A closed-loop theory of motor learning. Journal of Motor Behavior, 1971, 3, 111-150.

Alden, G. L., Siegel, A. W., \& Rosinski, R. R. The role of perceptual context in structuring spatial knowledge. Journal of Experimental Psychology: Human Learning and Memory, 1978, 4, 617-630.

Attneave, F., \& Benson, B. Spatial coding of tactile stimulation. Journal of Experimental Psychology, 1968, 81, 216-222.

Bremmer, J. G., \& Bryant, P. E. Place versus response as the basis of spatial errors made by using infants. Journal of Experimental Child Psychology, 1977, 23, 162-171.

Buck, L. The sensory basis of target location. Quarterly Journal of Experimental Psychology, 1979, 31, 111-120.

Burgess, P. R., \& Clark, F. J. Characteristics of knee joint receptors in the cat. Journal of Physiology (London), 1969, 203, 317-335.

DiEwERT, G. L. The role of vision and kinesthetics in coding of two-dimensional movement information. Journal of Human Movement Studies, 1976, 3, 191-198.

Gross, Y., Webs, R., \& Melzack, R. Central and peripheral contributions to localization of body parts: Evidence for a central body schema. Experimental Neurology, 1974, 44, 346-362.

Hart, R. A., \& Moore, G. The development of spatial cognition: A review. In R. M. Downs \& D. Stea (Eds.), Image and environment. Chicago: Aldine, 1973.

HollingwORTH, H. L. The inaccuracy of movement. Archives of Psychology, 1909, 2, 1-87.

Holmes, G. Disturbances of visual orientation. British Journal of Ophthalmology, 1919, 2, 449-468.

Howahd, I. P., \& Templeton, W. B. Human spatial orientation. London: Wiley, 1966.

Larish, D. D., Stelmach, G. E., \& McCracken, H. D. The generalizability of preselection. In G. C. Roberts \& K. M. Newell (Eds.), Psychology of motor behavior and sport-1978. Champaign, Ill: Human Kinetics Publishers, 1979.

LASHLEY, $\mathbf{K}$. S. The problem of serial order behavior. In $\mathbf{L}$. A. Jeffress (Ed.), Cerebral mechanisms in behavior. New York: Wiley, 1951.

LuriA, A. R. Human brain and psychological processes. New York: Harper and Row, 1966.

MacNeilage, P. F. Motor control and serial ordering of speech. Psychological Review, 1970, 77, 183-196.

MatThews, P. B. C. Mammalian muscle receptors and their central actions. London: Arnold, 1972.
Monasso, P. Spatial control of arm movements. Experimental Brain Research, 1981, 42, 223-227.

Nelson, T. O., \& Chaikuin, S. Immediate memory for spatial location. Journal of Experimental Psychology: Human Learning and Memory, 1980, 6, 529-545.

Paillard, J., \& Brouchon, M. Active and passive movements in the calibration of position sense. In S. J. Freedman (Ed.), The neuropsychology of spatially oriented behavior. Homewood, Ill: Dorsey Press, 1968.

Pick, H. L. Systems of perceptual and perceptual-motor development. In J. P. Hill (Ed.), Minnesota symposia on child psychology (Vol. 4). Minneapolis: University of Minnesota Press, 1970.

Pick, H. L., Yonas, A., \& Riesen, J. J. Spatial reference systems in perceptual development. In M. H. Bernstein \& W. Kesson (Eds.), Psychological development from infancy. Hillsdale, N.J: Erlbaum, 1979.

RIDDoch, G. Dissociation of visual perception due to occipital injuries, with special reference to appreciation of movement. Brain, 1917, 40, 15-57.

RIEser, J. J., \& PICK, H. L. Reference systems and the perception of tactual and haptic orientation. Perception \& Psychophysics, 1976, 19, 117-121.

Russell, D. G. Spatial location cues and movement reproduction. In G. E. Stelmach (Ed.), Motor control: Issues and trends. New York: Academic Press, 1976.

Sadalla, E. K., Burroughs, W. J., \& Staplin, L. J. Reference points in spatial cognition. Journal of Experimental Psychology: Human Learning and Memory, 1980, 6, 516-528.

Safrit, M. J., Spray, J. A., \& Diewert, G. L. Methodological issues in short-term motor memory research. Journal of Motor Behavior, 1980, 12, 13-28.

SheA, J. B. Effects of labelling on motor short-term memory. Journal of Experimental Psychology: Human Learning and Memory, 1977, 3, 92-99.

Wallace, S. A. The coding of location: $A$ test of the target hypothesis. Unpublished doctoral dissertation, University of Wisconsin-Madison, 1976.

Wallace, S. A. The coding of location: A test of the target hypothesis. Journal of Motor Behavior, 1977, 9, 157-169.

Winther, K. T., \& Thomas, J. R. Developmental differences in children's labelling of movement. Journal of Motor Behavior, 1981, 13, 77-90.

Woodworth, R. S. Accuracy of voluntary movement. Psychological Review Monograph Supplement, 1899, 3, 1-114.

\section{NOTES}

1. The vertical and horizontal tasks were originally conducted as independent experiments and are reported collectively because their procedures, design, and results are similar.

2. Since the criterion movement is constant between the intraand interlimb conditions, it might be argued that limb dominance was confounded with the interlimb condition. Thus, if differences are found between the two limb conditions, they could be attributed to the subjects' having less accuracy with their left arms. Wallace (1977), however, presented data that argue against this position, at least for movements in one dimension. He showed that reproduction errors in the right-right and left-left conditions were equivalent and reproduction errors in the right-left and leftright conditions were also equivalent. These findings indicate that reproduction accuracy is no better with one arm than the other. Results that reinforce this conclusion are presented in Experiment 2 of this study.

(Manuscript received September 23, 1980; revision accepted for publication April 6, 1982.) 